REVIEW ARTICLE

\title{
A review of functional and structural neuroimaging studies to investigate the inner speech model of auditory verbal hallucinations in schizophrenia
}

\author{
Liam Barber (iD ${ }^{1 凶}$, Renate Reniers ${ }^{2,3,4}$ and Rachel Upthegrove (iD) $3,4,5$
}

(c) The Author(s) 2021

\begin{abstract}
Although the pathophysiology of auditory verbal hallucinations remains uncertain, the inner speech model remains a prominent theory. A systematic review and meta-analyses of both functional and structural neuroimaging studies were performed to investigate the inner speech model. Of the 417 papers retrieved, 26 met the inclusion criteria. Meta-analyses found the left insula to be significantly active during auditory verbal hallucinations and to have a significantly reduced grey matter volume in hallucinators. Dysfunction of the left insula may contribute to the misattribution of inner speech due to its suggested roles in both inner speech production and the salience network. No significant activity was found at Broca's area or Heschl's gyrus during auditory verbal hallucinations. Furthermore, no structural abnormalities were found at these sites or in the arcuate fasciculi. Overall, evidence was found to both support and oppose the inner speech model. Further research should particularly include a systematic review of taskbased trait studies with a focus on inner speech production and self-referential processing, and analyses of additional languagerelated white matter tracts.
\end{abstract}

Translational Psychiatry (2021)11:582; https://doi.org/10.1038/s41398-021-01670-7

\section{INTRODUCTION}

Auditory verbal hallucinations (AVHs) are a distressing symptom of psychotic disorders [1], experienced as the perception of a voice in the absence of an external stimulus $[2,3]$. Of those with schizophrenia, $60-90 \%$ experience AVHs [4]; around 25\% of these have AVHs that are resistant to anti-psychotic medication [5]. AVHs are usually accompanied by an impairment in social and occupational functioning [6], and they are a significant risk factor for completed suicide [7].

The precise pathophysiology of $\mathrm{AVHs}$ is yet undetermined. However, growing evidence suggests a number of structural and functional changes within the brain, in keeping with prominent theories of AVH aetiology, including: (1) hyperexcitability of the auditory cortex with reduced top-down inhibition; (2) intrusive memories; and (3) the misattribution of inner speech to an external source $[8,9]$. The latter has seen a considerable advance in research in recent years, such that a review is warranted.

Inner speech is the process of covertly speaking to oneself to fulfil functions such as planning, verbal rehearsal and selfregulation [10]. It has been proposed that inner speech develops in childhood. Initially, children only speak overtly, in dialogues with others and with themselves; when overtly speaking to themselves, children instruct their own behaviour. Inner speech develops when this overt, self-directed speech is internalised [11].

Neuroimaging studies have highlighted areas in the brain that may be responsible for the production and monitoring of inner speech. A strong body of evidence supports the role of the left inferior frontal gyrus, including Broca's area, in the production of inner speech [10]. The left superior temporal gyrus includes the primary auditory cortex for speech perception (specifically, the primary auditory cortex is located in Heschl's gyrus) and Wernicke's area for speech comprehension. Additionally, the superior temporal gyri, as well as the anterior cingulate cortex and left inferior parietal lobule, have been found to contribute to self-referential processing [12] - which may be a key function in the successful monitoring of inner speech.

Corollary discharge dysfunction is one hypothesis used to explain how inner speech could be misattributed to an external source. It is proposed that the role of corollary discharge is to inform the auditory cortex that inner speech is being produced $[9,11,13]$. Subsequently, the activity of the auditory cortex decreases and inner speech is recognised as self-generated. Significant activity during AVHs in both the left inferior frontal gyrus and the left superior temporal gyrus may demonstrate corollary discharge dysfunction-leading to the misattribution of inner speech $[9,11]$. Supporting this hypothesis, in their metaanalyses, both Jardri et al. [5] and Kühn and Gallinat [14] found the left inferior frontal gyrus to be significantly active during AVHs in those with schizophrenia. However, only Jardri et al. [5] also found significant activity in the left superior temporal gyrus.

Also supporting corollary discharge dysfunction, Geoffroy et al. [15] found individuals with schizophrenia who experienced

\footnotetext{
${ }^{1}$ College of Medical and Dental Sciences, University of Birmingham, Edgbaston, Birmingham, UK. ${ }^{2}$ Institute of Clinical Sciences, University of Birmingham, Birmingham, UK. ${ }^{3}$ Centre for Human Brain Health, University of Birmingham, Birmingham, UK. ${ }^{4}$ Institute for Mental Health, University of Birmingham, Birmingham, UK. ${ }^{5}$ Early Intervention Service, Birmingham Women's and Children's NHS Trust, Birmingham, UK. ${ }^{凶}$ email: LDB498@alumni.bham.ac.uk
} 


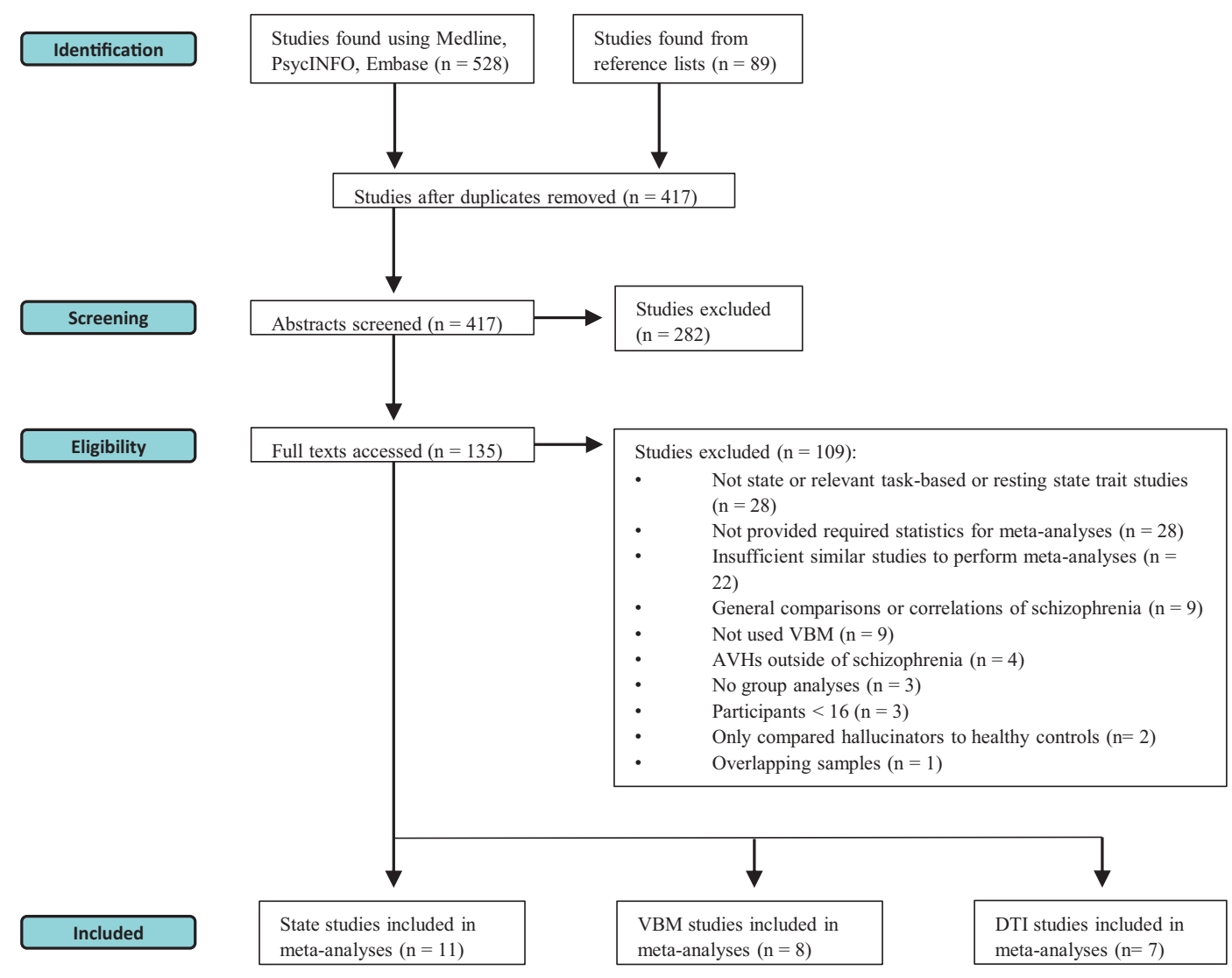

Fig. 1 A flow diagram illustrating the study selection process [51]. VBM voxel-based morphometry, AVHs auditory verbal hallucinations, DTI diffusion tensor imaging.

AVHs (hallucinators henceforth), had a significantly lower fractional anisotropy (FA) of the left arcuate fasciculus, compared to healthy controls. The left arcuate fasciculus connects the language centres of the frontal and temporal lobes and thus, is proposed as a pathway via which corollary discharge may travel [4]. However, in the absence of a comparison to individuals with schizophrenia who do not experience AVHs (non-hallucinators henceforth), a reduced FA could be a general feature of individuals with schizophrenia.

Despite the focus on the inner speech model here, some explain that the model does not explain the full complexity of AVHs. Particularly, this includes why individuals can experience $\mathrm{AVHs}$ in a range of different voices-rather than exclusively in their own voice [13]. Therefore, the aim of this systematic review is to collate and summarise the functional and neuroanatomical evidence of the inner speech model of AVHs. This will allow pooled results from the different modalities to be compared in one review, to identify congruence or conflict within the evidencebased. Overall, we aim to explore whether the current evidencebased supports the inner speech model of $\mathrm{AVHs}$.

\section{METHODS}

\section{Literature search and study selection}

The literature search was initially conducted in January 2018 and repeated in both August 2019 and August 2020 using the databases: Medline, PsycINFO and Embase. The search terms that were used to identify relevant functional neuroimaging studies were: "auditory hallucinat*" OR "auditory verbal hallucinat*" OR "verbal auditory hallucinat*" OR "verbal hallucinat" OR "hallucinat* spe*" OR "hear* voice*" OR "voice hear*" OR phoneme AND "inner spe ${ }^{* \prime}$ OR "internal* spe*" OR "covert spe*" OR "private spe ${ }^{* \prime}$
OR "subvocal* spe*" OR "internal dialogue" OR self-talk* OR monologue OR subvocali?* OR self-referen* OR "self-referential process $^{*}$ " OR self-monitor ${ }^{*}$ OR self-know* OR self-recogni* AND magnetic resonance imaging OR positron-emission tomography OR single photon emission computed tomography OR neuroimaging OR functional neuroimaging. The search terms that were used to identify relevant structural neuroimaging studies were: "auditory hallucinat*" OR "auditory verbal hallucinat*" OR "verbal auditory hallucinat" ${ }^{*}$ OR "verbal hallucinat ${ }^{*}$ " OR "hallucinat* spe $^{* "}$ OR "hear* voice*" OR "voice hear" tensor imaging OR "diffusion tensor" OR "diffusion weighted" OR "DWI" OR "diffusion weighted imaging" OR "fractional anisotropy" OR voxel-based OR voxelwise OR morphometry. The reference lists of relevant reviews and meta-analyses were hand-searched to identify studies that were missed by the database search. The selection of studies was not limited by publication date or by the language in which they were published.

The eligibilities of 417 studies were assessed, firstly, using their titles and abstracts. Secondly, the full texts of 135 studies were accessed. For a flow diagram illustrating the selection process (see Fig. 1). If the eligibility of a certain study was unclear, a second reviewer, at least, was consulted and a consensus was reached.

Inclusion criteria:

1. Studies included participants (aged 16 or older) diagnosed with schizophrenia, who experienced AVHs.

2. Functional neuroimaging studies that used functional magnetic resonance imaging, positron emission tomography or single-photon emission computed tomography.

3. Task-based trait studies that specifically used tasks involving inner speech production, auditory verbal imagery or voice recognition.

4. Structural neuroimaging studies that used DTI or VBM analysis. 


\section{Exclusion criteria:}

1. Case reports.

2. Intervention studies.

3. Studies that did not present primary data.

4. Studies that artificially induced AVHs.

5. Studies solely comparing hallucinators to healthy controls.

\section{Data extraction}

A standard table was created to support the extraction of required information from studies, including: sample size; characteristics of participants (age, sex, handedness, duration of illness, severity of AVHs, anti-psychotic medication dosage and length of use, history of substance misuse/dependence); imaging modality; data analysis method; stereotaxic coordinates (three-dimensional- $x, y, z$ ); means and standard deviations of DTI metrics (FA, mean diffusivity, radial diffusivity, axial diffusivity).

It was important to extract detailed data on the characteristics of participants to assess whether primary studies controlled for certain variables. The duration of illness and the use of antipsychotic medication are two variables that have been shown to cause structural changes in the brain [16]. Curčić-Blake et al. [9] found that the FA of white matter tracts was generally increased in participants with acute psychosis but decreased in those with chronic psychosis. Therefore, using this example, significant differences in the duration of illness between hallucinators and non-hallucinators could alter the results of the meta-analyses.

\section{Meta-analyses of state studies}

BrainMap's GingerALE 3.0.2 software was used to perform the meta-analyses of state studies. Specifically, the non-additive ALE method was used to limit the effect of multiple findings near to one another, on an individual study's overall contribution to a meta-analysis [17]. The smaller mask (of the coordinate space) was used as this is recommended for an ALE meta-analysis of functional studies [18].

The coordinates used in the meta-analyses were the locations of peak voxels of significant clusters reported in each included study. To allow inclusion in ALE meta-analyses, studies must present their coordinates in Talairach or Montreal Neurological Institute space. If a study presented its coordinates in Talairach space, the icbm2tal transform [19] was used to convert these into Montreal Neurological Institute space.

The sample size used corresponded to the size of the smallest group included in each study. This is the recommended and most conservative approach because there is more uncertainty regarding the precise location of a finding if a small sample is used [20].

The threshold for statistical significance was set using the cluster-level family-wise error (FWE) correction. This correction ensures the exclusion of small clusters that exceed the statistical threshold but have arisen due to chance. Moreover, it is more sensitive than the voxel-wise FWE correction [21]. Maps of statistically significant clusters were overlaid onto an anatomical template (Colin27_T1_seg_MNI.nii) using the Mango image viewer (rii.uthscsa.edu/mango) [22].

\section{Meta-analyses of trait studies}

Meta-analyses of both task-based trait studies and resting-state trait studies were planned. Regarding task-based trait studies, due to the focus on the inner speech model of AVHs, only studies that used tasks requiring inner speech production or self-referential processing were eligible. Furthermore, as different tasks have discrete cognitive requirements, only studies using the same task design were to be combined in a single meta-analysis. A metaanalysis of resting-state trait studies would also be relevant because inner speech production and self-referential processing are associated with wakeful rest [23]. However, no meta-analyses of trait studies (both task-based and resting-state) could be performed because an insufficient number of studies were found for each meta-analysis.

\section{Meta-analyses of voxel-based morphometry studies}

The method used to perform the meta-analyses of VBM studies was very similar to the method described above for state studies. The software used and the statistical threshold was the same. The one difference was that a larger mask of the coordinate space was used because this is preferable for a meta-analysis of structural studies-to limit the number of coordinates located outside of the brain [18].

Two meta-analyses of VBM studies were performed: (1) to identify regions where the grey matter volume was significantly reduced in hallucinators compared to non-hallucinators; (2) to identify regions where a reduction in grey matter volume was significantly associated with increased severity of hallucinations. As too few eligible studies were found, meta-analyses were not performed to identify regions where grey matter volume was significantly increased in hallucinators compared to non-hallucinators, nor where an increase in grey matter volume was significantly associated with increased severity of hallucinations.

\section{Meta-analyses of diffusion tensor imaging studies}

Review Manager 5.3 (RevMan) [24] was used to perform the metaanalyses of DTI studies. For each study, the sample size of each group and the mean and standard deviation of a DTI metric were entered into RevMan. The mean difference was then calculated with a $95 \%$ confidence interval. Accounting for the weight attributed to each study (an inverse variance method was used to combine the mean differences; thus, the weight of each study was equivalent to the reciprocal of its variance), the summary effect and its 95\% confidence interval were determined, and a forest plot was produced.

Ideally, meta-analyses would have been performed for a range of language-related tracts, using various DTI metrics. For relevant studies that did not present the required means and standard deviations, these were requested by contacting the corresponding authors. However, too few studies provided the essential statistics to perform meta-analyses for additional tracts. Moreover, too few studies measured the mean diffusivity, radial diffusivity or axial diffusivity; hence, meta-analyses using these DTI metrics were not performed. Therefore, two meta-analyses were performed to compare the FA of the left and right arcuate fasciculi between hallucinators and non-hallucinators. To account for multiple comparisons, a was divided by the number of meta-analyses performed [25] to give a corrected $p$-value $(p=0.025)$. The level of heterogeneity is represented by the $I[2]$ index; as $I^{2}>50 \%$ in both meta-analyses, a random-effects model was used because this is the more conservative approach $[25,26]$.

\section{RESULTS}

Meta-analyses of state studies

Table 1 summarises the characteristics of the state studies included in the meta-analysis. Two clusters were found to be significantly active during AVHs (see Fig. 2 and Table 2). One cluster was in the left hemisphere, which was centred in the inferior parietal lobule and also included the postcentral gyrus and the insula. The remaining cluster was in the right anterior lobe of the cerebellum, which was centred in the culmen and also included the dentate.

The meta-analysis of state studies was performed twice because the studies by Sommer et al. [27] and Diederen et al. [28] had overlapping samples. As both studies had equal sample sizes and used similar statistical thresholds, one study could not be favoured. Hence, a second meta-analysis of state studies was performed, including the study by Diederen et al. [28] rather than 
Table 1. The characteristics of the state studies included in the meta-analyses.

\begin{tabular}{|c|c|c|c|c|c|}
\hline Study & Imaging modality & No. of hallucinators & Whole-brain or ROI analysis & No. of foci & Stereotaxic space \\
\hline Silbersweig et al. [52] (1995) & PET & 5 & Whole-brain & 9 & Talairach \\
\hline Lennox et al. [53] (2000) & fMRI & 4 & Whole-brain & 4 & Talairach \\
\hline Shergill et al. [36] (2000) & fMRI & 5 & Whole-brain & 27 & Talairach \\
\hline Copolov et al. [54] (2003) & PET & 8 & Whole-brain & 6 & Talairach \\
\hline Hoffman et al. [55] (2008) & fMRI & 6 & Whole-brain & 2 & Talairach \\
\hline Sommer et al. [27] $(2008)^{a}$ & fMRI & 24 & Whole-brain & 21 & MNI \\
\hline Diederen et al. [57] (2013) & fMRI & 33 & ROI & 5 & MNI \\
\hline Horga et al. [58] (2014) & fMRI & 9 & Whole-brain & 1 & MNI \\
\hline Thoma et al. [59] (2016) & fMRI & 15 & Whole-brain & 9 & $\mathrm{MNI}$ \\
\hline
\end{tabular}

$P E T$ positron emission tomography, $f M R I$ functional magnetic resonance imaging, $R O I$ regions of interest, $M N I$ Montreal Neurological Institute.

${ }^{a}$ The sample of these studies overlapped thus were included in separate meta-analyses.

the Sommer et al. [27] study. In this second meta-analysis, the cluster in the left hemisphere was essentially replicated; the cluster in the right cerebellum was not found-and no additional clusters were found (see Fig. 2 and Table 3).

\section{Meta-analyses of voxel-based morphometry studies}

Table 4 summarises the characteristics of the VBM studies included in the meta-analyses. Neither the meta-analysis comparing hallucinators to non-hallucinators nor the meta-analysis looking for significant associations between grey matter volume reduction and hallucination severity produced significant findings.

A subgroup analysis was performed with a focus on how nonhallucinators were defined, specifically if they had ever experienced AVHs-rather than not currently experiencing AVHs-as this could reduce the chance of identifying significant differences in brain structure. Hallucinators were compared to non-hallucinators, with two studies excluded from this analysis. Again, no significant clusters were found. The study by Cierpka et al. [29] was excluded because 8 of the 10 non-hallucinators they included had experienced $\mathrm{AVHs}$ - but not in the previous 12 months. The study by Shapleske et al. [30] was excluded because they defined nonhallucinators by a score of $<2$ (for all but 1 week of their illness) on the auditory hallucination item of the Scale for Assessment of Positive Symptoms (SAPS). A score of 0 would mean AVHs were absent in this group of non-hallucinators; a score of 1 means these non-hallucinators may have experienced AVHs.

A significant cluster was found by a sensitivity analysis. In the sensitivity analysis, only studies that adequately controlled for additional variables (minus the presence of AVHs) were included. Thus, in this sensitivity analysis, the study by Gaser et al. [31] was excluded because no evidence of control for age, duration of illness or use of antipsychotics was found. The result was a cluster in the left hemisphere, centred in the claustrum and also included the putamen and insula, where hallucinators had a significantly reduced grey matter volume compared to non-hallucinators (see Fig. 3 and Table 5).

\section{Meta-analyses of diffusion tensor imaging studies}

Table 6 summarises the characteristics of the DTI studies included in the meta-analyses. For the FA of the left arcuate fasciculus, no significant difference was found between hallucinators and nonhallucinators (0.00 [ -0.02 to 0.01$] ; p=0.71$; see Fig. 4). Similarly, for the FA of the right arcuate fasciculus, no significant difference was found between hallucinators and non-hallucinators $(-0.01$ [ -0.03 to 0.01$] ; p=0.4$; see Fig. 5 ).

Subgroup analyses were also performed for both the left and right arcuate fasciculi - focusing on the definition of non-hallucinators.
These subgroup analyses also did not produce significant results. The study by Xie et al. [32] was excluded because they defined nonhallucinators by a score of $\leq 2$ on the hallucinatory behaviour item (P3) of the positive and negative syndrome scale (PANSS). A score of 2 describes minimal hallucinatory behaviour-although the modality in which the hallucinatory behaviour occurred was not specified.

Likewise, when sensitivity analyses were performed for each tract, the results did not reach significance. The studies by Leroux et al. [33] and Chawla et al. [34] were excluded. This was because the Leroux et al. [33] study found hallucinators were taking a significantly higher dose of antipsychotics than non-hallucinators. The Chawla et al. [34] study found hallucinators had a significantly longer duration of illness than non-hallucinators.

\section{DISCUSSION}

Several meta-analyses were performed in this systematic review to investigate the inner speech model of AVHs. The centres for inner speech production and perception were not found to be significantly active by the meta-analyses of state studies. Moreover, in meta-analyses comparing hallucinators to non-hallucinators, significant structural abnormalities were not found in these centres or in the arcuate fasciculi. These negative findings do not support the inner speech model of AVHs. However, the role of the left insula requires further consideration because it was found to be both significantly active during $\mathrm{AVH}$, and to have a significantly reduced grey matter volume in hallucinators compared to non-hallucinators.

\section{Cerebral activity during auditory verbal hallucinations}

Unlike the meta-analyses by Jardri et al. [5] and Kühn and Gallinat [14], the left inferior frontal gyrus was not found to be significantly active in either meta-analyses of state studies presented here. The absence of significant activity in the left inferior frontal gyrus seems to preclude inner speech production. However, the left inferior frontal gyrus may be predominantly active prior to the experience of an $\mathrm{AVH}$-causing its activity to go undetected by some state studies [35]. Alternatively, the left insula has been found to be significantly active during inner speech production $[36,37]$. The left insula was found to be significantly active in both meta-analyses of state studies here, and that by Jardri et al. [5]. Thus, for inner speech production, significant activity in either the left inferior frontal gyrus or the left insula may be required-rather than in both. A meta-analysis of inner speech production in healthy volunteers would prove useful when evaluating the inner speech model of $\mathrm{AVHs}$, if the meta-analysis clarified the regions involved in inner speech production. 
a

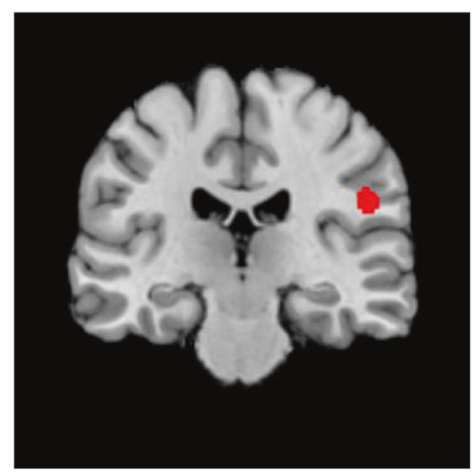

b

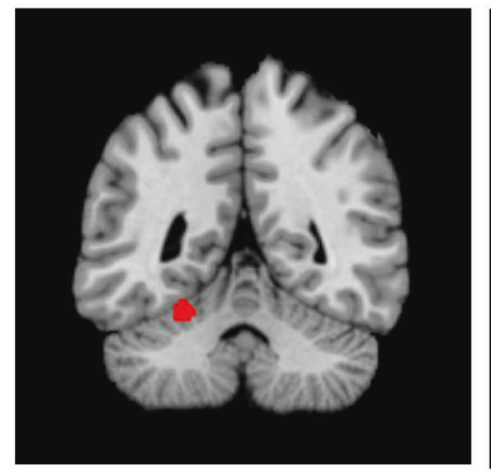

C

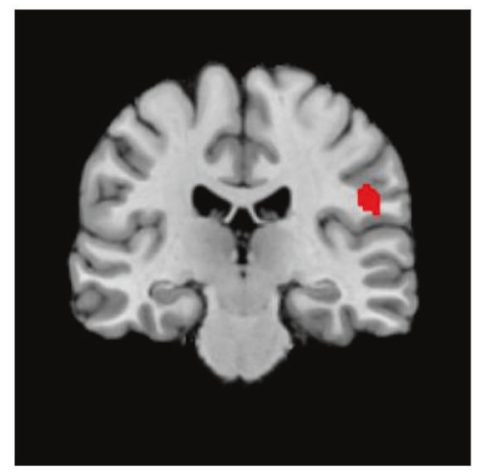

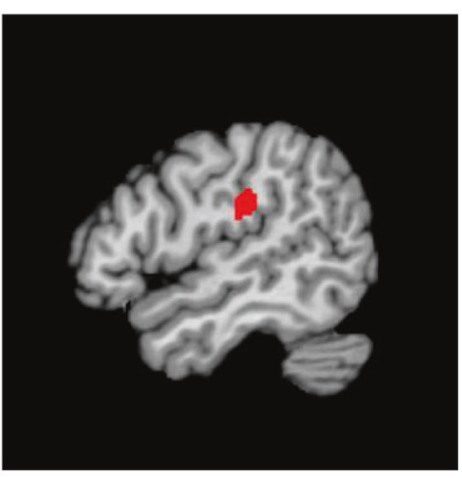
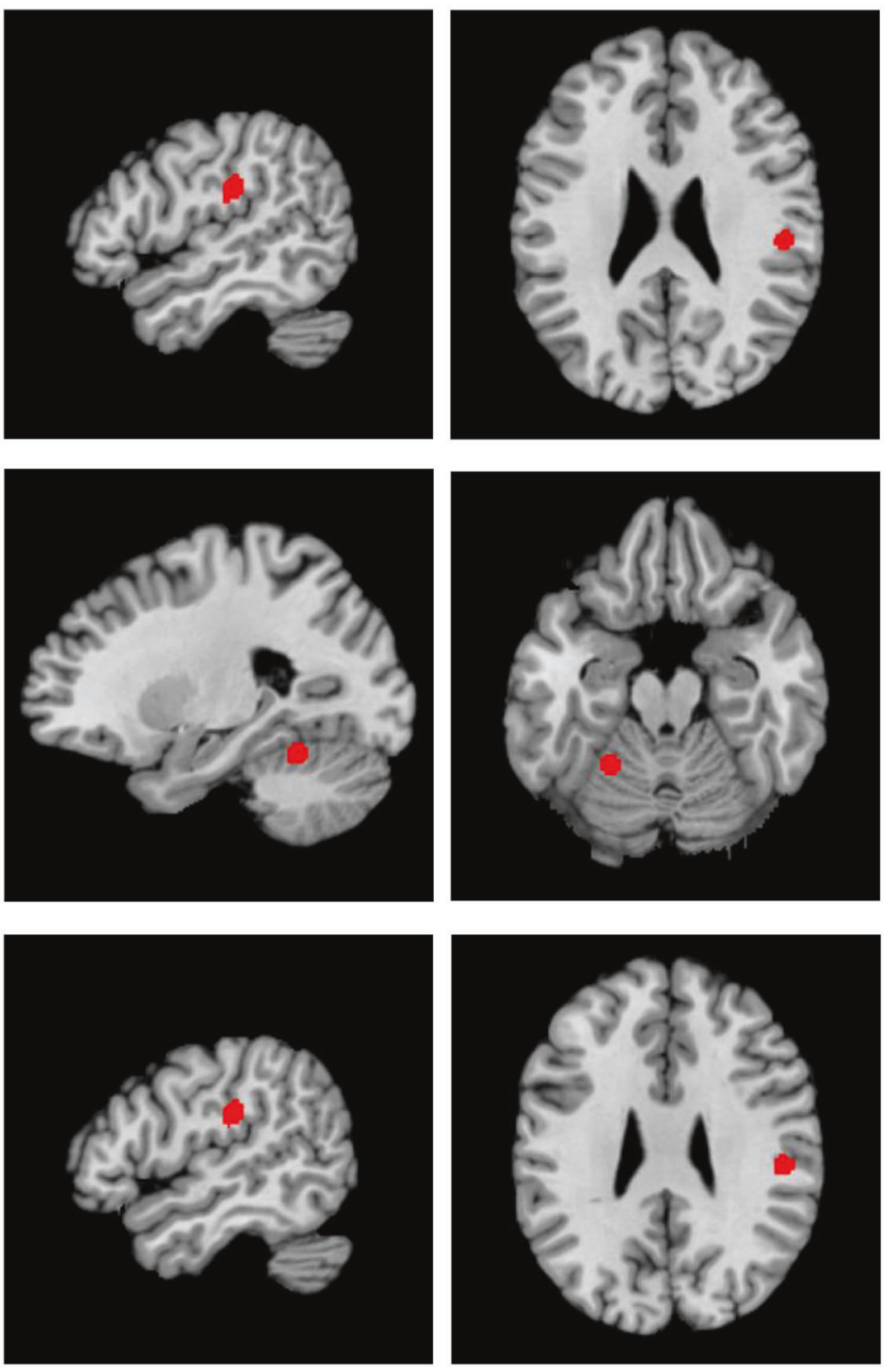

Fig. 2 The location of the clusters of significant activity during auditory verbal hallucinations (AVHs), found by the meta-analyses of state studies. The location of the significant clusters, in coronal (left), sagittal (middle) and axial (right) planes, are highlighted in red. a The significant cluster in the left hemisphere, centred in the inferior parietal lobule and also including the postcentral gyrus and the insula. $\mathbf{b}$ The significant cluster in the right anterior lobe of the cerebellum, centred in the culmen and also including the dentate. c The result of the second meta-analysis of state studies (which included the Diederen et al. [28] study rather than the Sommer et al. [27] study) finding a significant cluster in the left hemisphere, centred in the inferior parietal lobule and also including the postcentral gyrus and the insula.

In addition to the proposed role of the left insula in inner speech production, the insula and the anterior cingulate cortex form the salience network. The significant activity of the left insula in the meta-analyses of state studies may demonstrate activity in the salience network during AVHs. If excess salience is attributed to inner speech, this may increase the likelihood of misattributing inner speech to an external source [38].

The lack of significant activity in the left superior temporal gyrus seems to preclude inner speech perception; however, the absence of activity in the superior temporal gyri is also relevant when considering the efficacy of self-referential processing in hallucinators. Of the regions $\mathrm{Hu}$ et al. [12] found to be involved in selfreferential processing, only the left inferior parietal lobule was found to be significantly active in the meta-analyses of state studies here. Furthermore, during a working memory task that required inner speech for rehearsal, Wible et al. [39] found hallucinators had significantly reduced activity in the left inferior parietal lobule and left superior temporal gyrus when compared to non-hallucinators. Thus, the absence of significant activity in many regions involved in self-referential processing may demonstrate a disorder of self-referential processing in hallucinators; activity in the left inferior parietal lobule alone may be insufficient for effective self-referential processing. To explore this further, when a sufficient number of studies are available, it will be useful to perform the meta-analyses of task-based trait studies suggested above. This will provide a more robust comparison of self-referential processing between hallucinators and nonhallucinators.

Although it has been suggested that the cerebellum may also have a role in speech production [29, 40], an alternative explanation is that the cluster of significant activity in the right anterior lobe of the cerebellum, centred in the culmen and extending to include the dentate, is related to the movement involved in indicating the onset of an $\mathrm{AVH}$. This also applies to the significant activity found in the post-central gyrus. Furthermore, the cluster in the cerebellum was not found when the meta- 

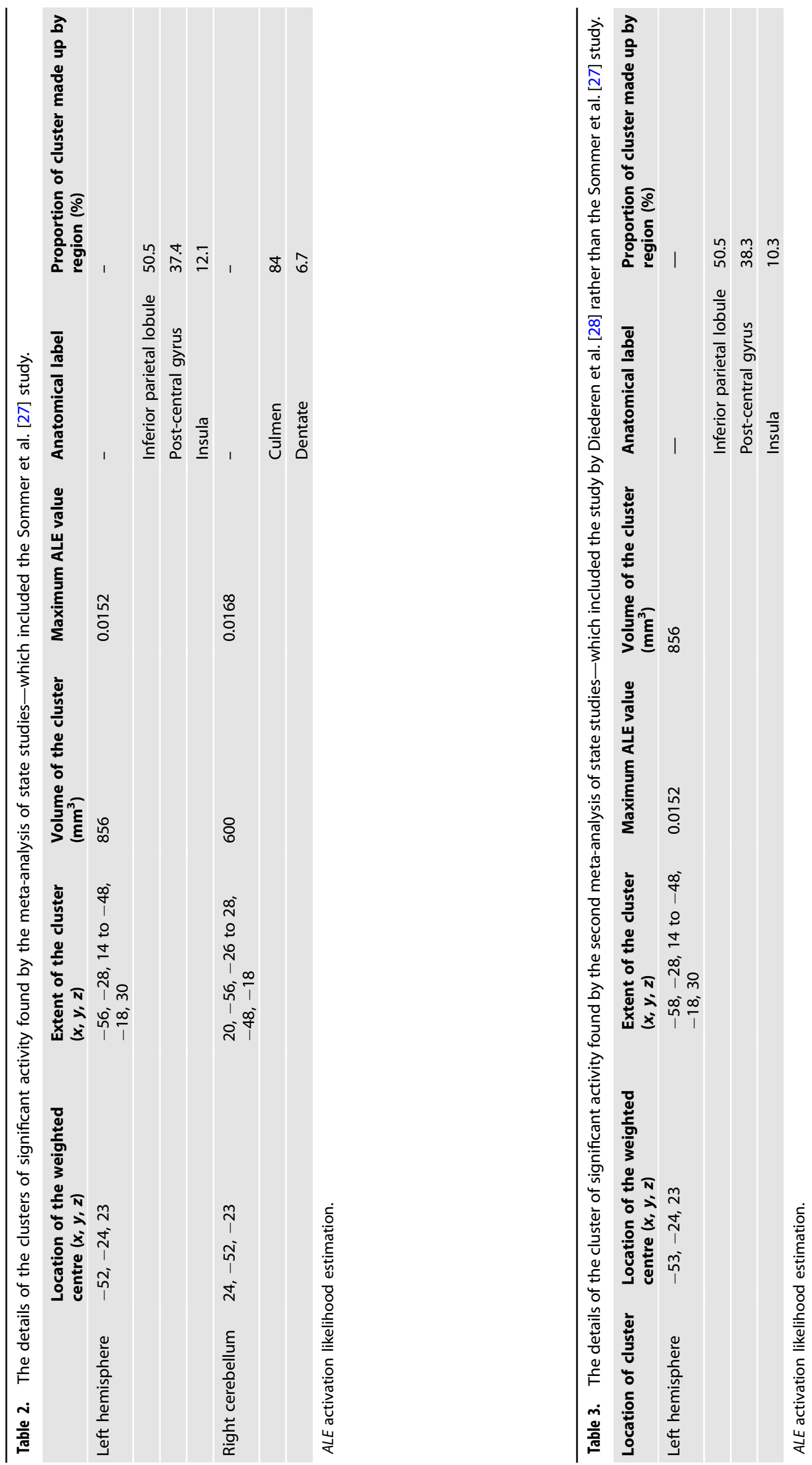


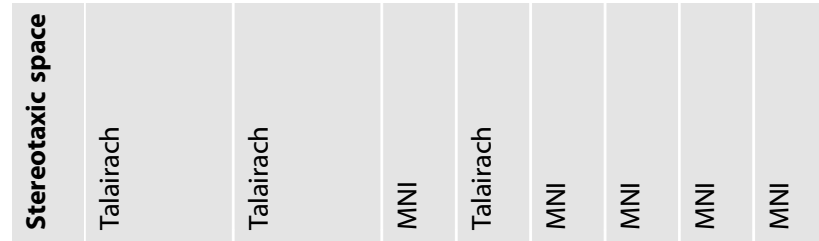
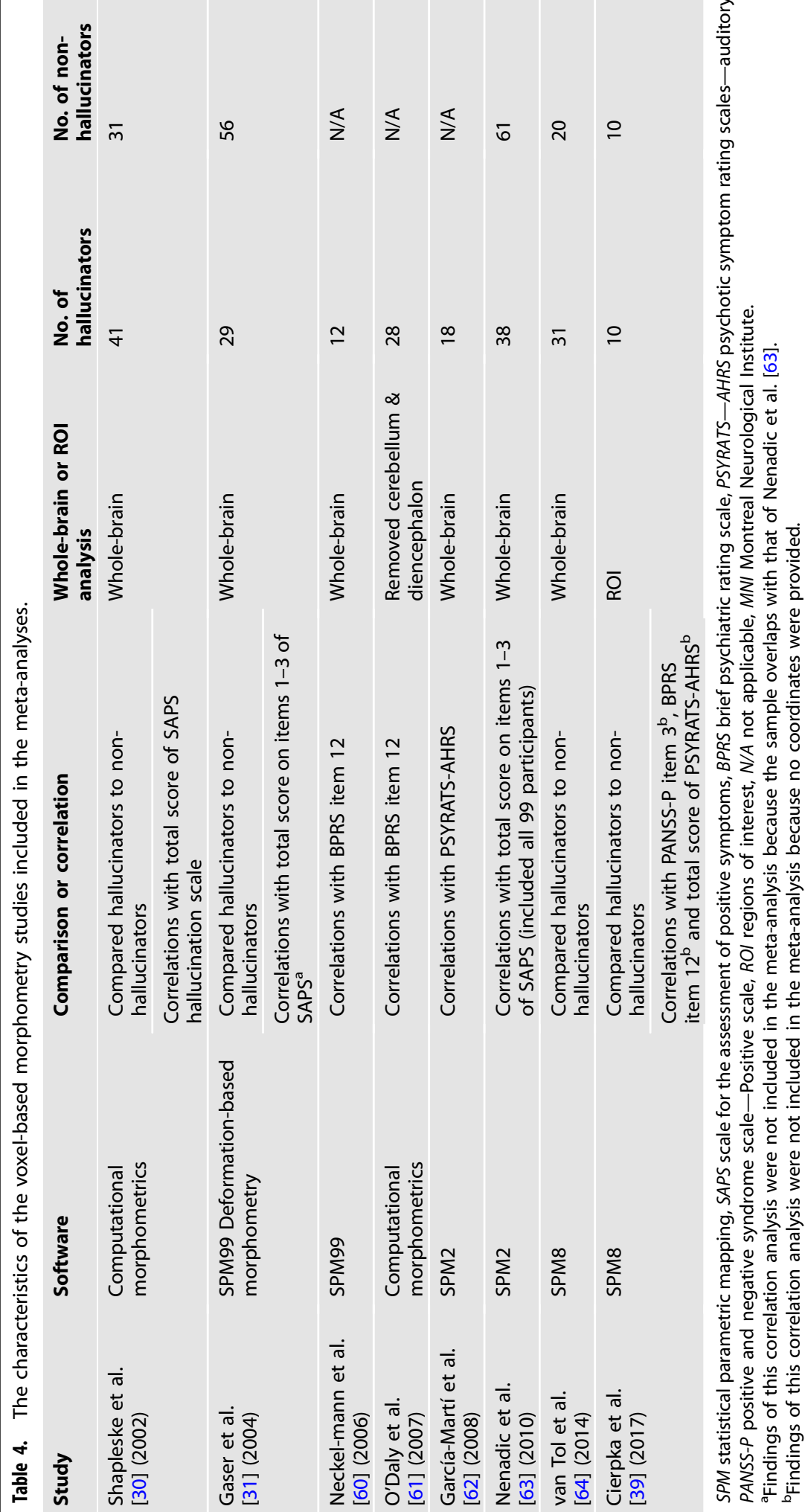

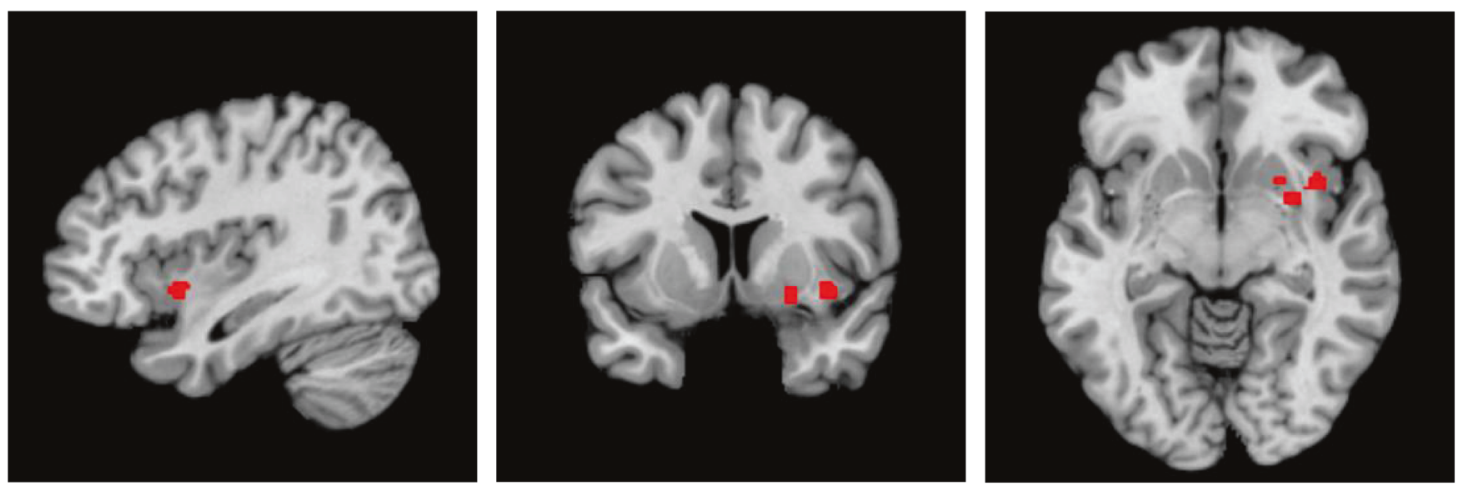

Fig. 3 The result of the sensitivity analysis of voxel-based morphometry studies showing the location of the cluster of significant grey matter volume reduction in the left hemisphere, centred in the claustrum and also including the putamen and insula, in hallucinators compared to non-hallucinators. The location of the significant cluster, in sagittal (left), coronal (middle) and axial (right) planes, are highlighted in red.

analysis was repeated using the Diederen et al. [28] study as opposed to the Sommer et al. [27] study.

There are numerous reasons for differences in results between the meta-analyses of state studies here and those by Jardri et al. [5] and Kühn and Gallinat [14]. Importantly, several additional studies were included here simply because this is an up-to-date review of the evidence-base. Furthermore, both Jardri et al. [5] and Kühn and Gallinat [14] used the false discovery rate to correct for multiple comparisons; the more stringent cluster-level FWE correction was used here.

Overall, the meta-analyses of state studies performed here do not clearly support the inner speech model of AVHs. Predominantly, this is because significant activity was not found in the left inferior frontal gyrus or the left superior temporal gyrus. However, the current findings highlight that in addition to the left inferior frontal gyrus, the insula and cerebellum may be involved in inner speech production. Furthermore, the meta-analyses suggest that self-referential processing is impaired in hallucinators. Subsequently, hallucinators may have difficulty when determining whether an auditory stimulus is self-generated, making it possible to misattribute inner speech to an external source.

\section{Regional grey matter volume reductions in hallucinators}

Alongside the meta-analyses of state studies presented here, the sensitivity analysis of VBM studies reinforces the importance of the insula in the pathophysiology of AVHs. The sensitivity analysis found the left insula formed part of a cluster, centred in the claustrum and also including the putamen, where there was a significantly reduced grey matter volume in hallucinators compared to non-hallucinators. Consistent with these findings, in their meta-analysis of VBM studies, Palaniyappan et al. [8] found both the left and right insula were included in clusters where a reduced grey matter volume was significantly linked to increased hallucination severity.

The role the claustrum and putamen may have in the pathophysiology of $\mathrm{AVHs}$ is currently speculative. A reduced grey matter volume of the left claustrum, along with the right insula, has been significantly correlated with positive symptoms-but particularly with delusions, rather than AVHs [41]. The claustrum is a highly connected region that may serve as a hub to coordinate activity of cerebral circuits by gating selective attention [42, 43]. Like the proposed role of the salience network in the pathophysiology of AVHs, dysfunction of the left claustrum may contribute to AVHs by failing to direct attention away from inner speech. However, the evidence on how the claustrum could contribute to the misattribution of inner speech is not robust-warranting further investigation. Regarding the putamen, this region has been linked to speech. However, the putamen appears to be involved in vocalisation as opposed to word selection and sentence production [44, 45].

\section{The integrity of white matter tracts in hallucinators}

Interpreting the results of the meta-analyses of DTI studies here, alongside those by Geoffroy et al. [15], suggests a reduced FA of the left arcuate fasciculus is a general abnormality of schizophrenia-rather than specific to hallucinators. This is because Geoffroy et al. [15] found hallucinators had a significantly reduced FA of the left arcuate fasciculus when compared to healthy controls; here, no significant differences were found between hallucinators and non-hallucinators for the FA of either the left or right arcuate fasciculi. However, Geoffroy et al. [15] used a fixed effects model despite high heterogeneity which, they explain, should be done when a meta-analysis consists of a small number of studies. Yet, a random effects model was preferred here to adopt the most conservative approach.

More primary DTI studies and meta-analyses are required to continue to investigate the pathophysiology of AVHs. Rather than disregarding the importance of the arcuate fasciculi, should additional studies become available, it would be worth repeating the above meta-analyses. Additionally, meta-analyses of other language-related tracts, using more DTI metrics where possible, would be informative.

When considering the findings of the meta-analysis of state studies, it may be useful to investigate the connectivity of the left inferior parietal lobule. The two short segments of the left arcuate fasciculus connect Broca's area and Wernicke's area via the left inferior parietal lobule [34]. Poor integrity, specifically along these short segments, could impair the communication between the left inferior parietal lobule and the language centres, hence, impairing self-referential processing. If the left insula has a role in inner speech production, it may also be useful to investigate the integrity of the white matter tracts that connect the left insula to the language centres and to the left inferior parietal lobule.

It is valuable to use multiple DTI metrics rather than the FA alone because together they allow a more detailed interpretation of findings. A reduced FA can be due to a decrease in the number, size or organisation of axons, a reduced integrity of their axolemma, or demyelination $[1,46]$. Whereas, for example, a high radial diffusivity suggests demyelination [1, 47]. Therefore, if a reduced $F A$ and an increased radial diffusivity are found together, it is more likely that the primary abnormality of that tract is of the myelin sheath. This would have implications for the inner speech model because damage to the myelin sheath could cause conduction delays. As in the theory of dysfunctional corollary discharge, if conduction is delayed in tracts carrying information to the auditory cortex, signals may arrive too late, leading to an increased auditory cortex activity despite the perception of inner speech. 


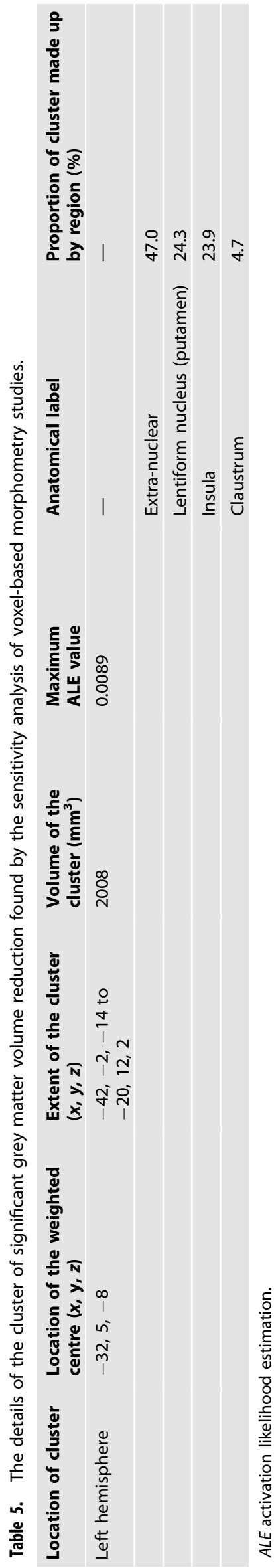

\section{Limitations}

The meta-analyses presented in this systematic review have several strengths, such as: using stringent statistical thresholds; comparing hallucinators to non-hallucinators; and considering the effects of confounding variables. However, there are some limitations. Firstly, studies using a regions of interest analyses were not excluded; this can cause the meta-analyses to become biased towards certain brain regions [3]. It was anticipated that, due to the existence of the inner speech model, some studies would use the areas involved in speech production, speech perception and self-referential processing as regions of interest. To assimilate the evidence to fully explore the inner speech model of $\mathrm{AVHs}$, it was deemed essential to also include these studies.

Secondly, rather than using ALE, it would have been optimal to perform image-based meta-analyses. However, this would have required the full imaging datasets of each included study-but these are not readily available. Therefore, ALE was chosen as it is the coordinate-based meta-analysis method that has been shown to produce findings that are most comparable to those produced by image-based meta-analyses [48]. Yet only the coordinates of significant findings from primary studies were used for our ALE meta-analyses - which is routine. This means that certain regions, where subtle changes contribute to the development of AVHs, causing only a trend to be found in primary studies, may remain undiscovered unless a transition to image-based meta-analysis is made. Furthermore, Eickhoff et al. [49] explained that for an ALE meta-analysis, 30 studies need to be included to have $80 \%$ power to find a region to be significant if that region is individually identified by $20 \%$ of the included studies. Therefore, if ALE is to be used, more state and VBM studies are required for greater power to detect significant clusters.

Finally, in sensitivity analyses, studies were excluded if the use of anti-psychotics was significantly different between hallucinators and non-hallucinators, given severity of illness (and hence persistent hallucinations) could be confounded by higher antipsychotic dose, and there is the potential for medication to effect brain structure [50]. Nevertheless, if anti-psychotics had induced remission in those defined as hallucinators, structural brain changes contributing to the experience of AVHs may no longer be present. Yet, in the DTI metaanalyses, 223 out of the 267 hallucinators had ongoing AVHs at the time of scanning - the majority despite anti-psychotic medication. Therefore, if structural brain changes are related to AVHs, these effects should have persisted in the majority of hallucinatorsirrespective of anti-psychotic use. The numbers are less clear for the VBM studies. However, at least 41 out of the 111 hallucinators included in the comparison to non-hallucinators had ongoing AVHs at the time of scanning-again, the majority despite anti-psychotic medication. To minimise the risk of anti-psychotics affecting structural neuroimaging findings, comparing medication naïve hallucinators and non-hallucinators would be optimal-yet challenging, as effective treatment cannot be delayed to acquire such data. Thus, comparing hallucinators (with active AVHs) and non-hallucinations, who both have minimal antipsychotic exposure may be the most pragmatic approach.

\section{CONCLUSION}

The aim of this systematic review was to investigate the inner speech model of AVHs, using up-to-date meta-analyses of both functional and structural neuroimaging studies, to identify whether the current evidence-base supports this model. The findings were mixed. For the centres of inner speech production and perception, no significant activity was found during AVHs. Furthermore, no significant structural abnormalities were found when hallucinators were compared to non-hallucinators in either of these areas, or in the arcuate fasciculi. These negative findings oppose the inner speech model. However, the left insula was found to be both significantly active during AVHs, and to have a 
Table 6. The characteristics of the diffusion tensor imaging studies included in the meta-analyses.

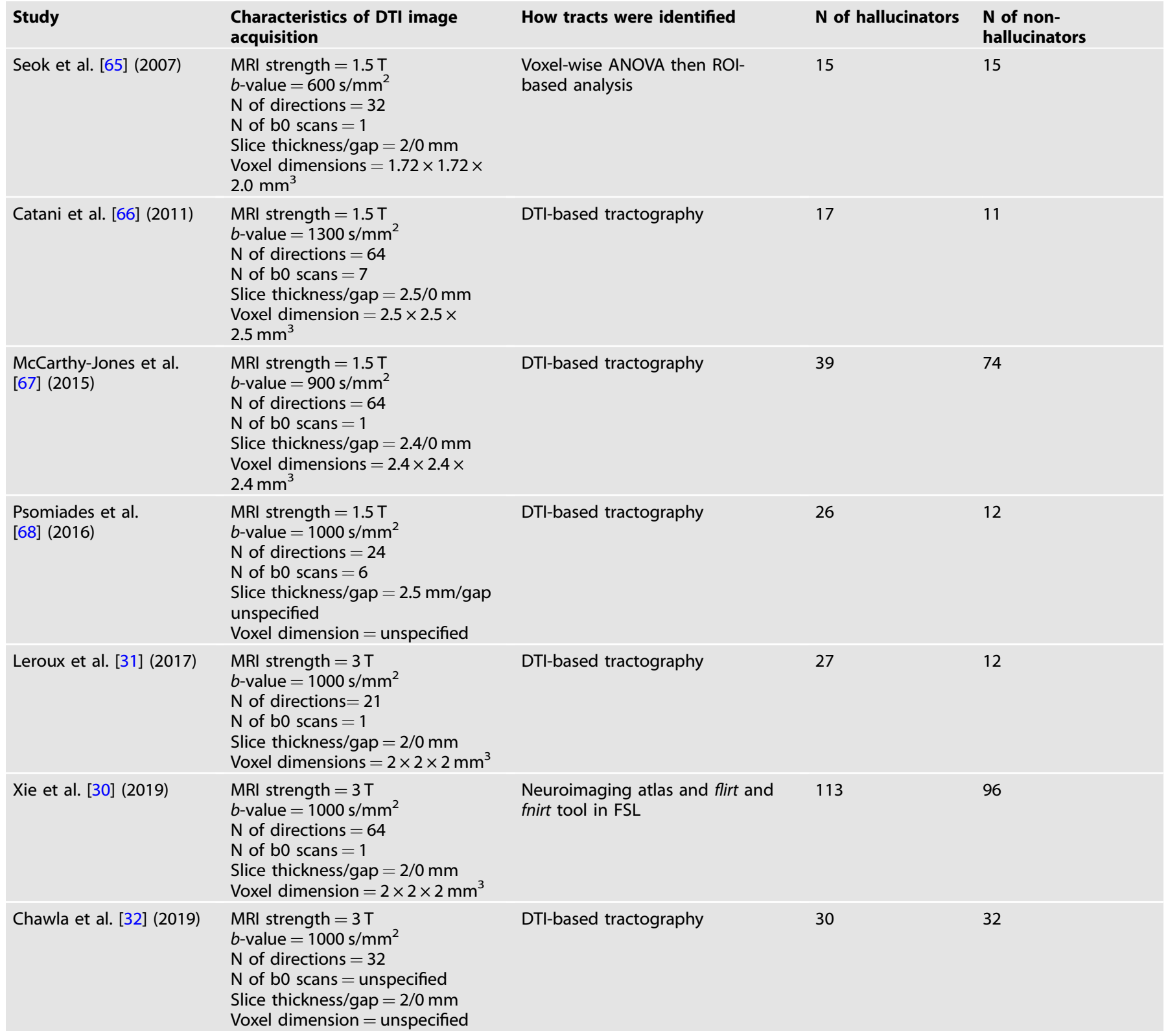

$D T I$ diffusion tensor imaging, MRI magnetic resonance imaging, $T$ Tesla, $b$ diffusion weighting, $N$ number, $A N O V A$ analysis of variance, $R O I$ region of interest, $F S L$ FMRIB software library.

\begin{tabular}{|c|c|c|c|c|c|c|c|c|c|}
\hline \multirow[b]{2}{*}{ Study or Subgroup } & \multicolumn{3}{|c|}{ Hallucinators } & \multicolumn{3}{|c|}{ Non-hallucinators } & \multicolumn{3}{|c|}{ Mean Difference } \\
\hline & Mean & SD & Total & Mean & SD & Total & Weight & IV, Random, $95 \% \mathrm{CI}$ & Year \\
\hline Seok 2007 & 0.26 & 0.02 & 15 & 0.23 & 0.03 & 15 & $13.0 \%$ & $0.03[0.01,0.05]$ & 2007 \\
\hline Catani 2011 & 0.416 & 0.02 & 17 & 0.424 & 0.015 & 11 & $14.4 \%$ & $.00]$ & 2011 \\
\hline McCarthy-Jones 2015 & 0.5026 & 0.0254 & 39 & 0.5219 & 0.0284 & 74 & $15.0 \%$ & $.01]$ & 2015 \\
\hline Psomiades 2016 & 0.434 & 0.019 & 26 & 0.417 & 0.019 & 12 & $14.4 \%$ & $.03]$ & 2016 \\
\hline Leroux 2017 & 0.475 & 0.0299 & 27 & 0.482 & 0.0191 & 12 & $13.7 \%$ & $-0.01[-0.02,0.01]$ & 2017 \\
\hline Xie 2019 & 0.471 & 0.033 & 113 & 0.465 & 0.033 & 96 & $15.3 \%$ & $, 0.01]$ & 2019 \\
\hline Chawla 2019 & 0.4935 & 0.0254 & 30 & 0.5289 & 0.0298 & 32 & $14.2 \%$ & $-0.04[-0.05,-0.02]$ & 2019 \\
\hline Total $(95 \% \mathrm{Cl})$ & & & 267 & & & 252 & $100.0 \%$ & $-0.00[-0.02,0.01]$ & \\
\hline
\end{tabular}

Heterogeneity: $\mathrm{Tau}^{2}=0.00 ; \mathrm{Chi}^{2}=57.19, \mathrm{df}=6(\mathrm{P}<0.00001) ; \mathrm{I}^{2}=90 \%$

Test for overall effect: $Z=0.37(P=0.71)$

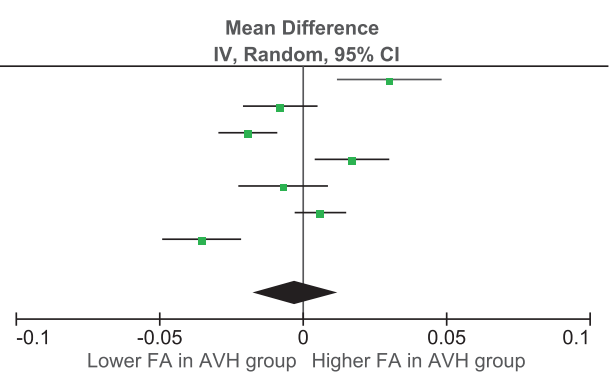

Fig. 4 A forest plot showing no significant difference between hallucinators and non-hallucinators for the fractional anisotropy of the left arcuate fasciculus. $S D$ standard deviation, $I V$ inverse variance, $C I$ confidence interval, $F A$ fractional anisotropy. 


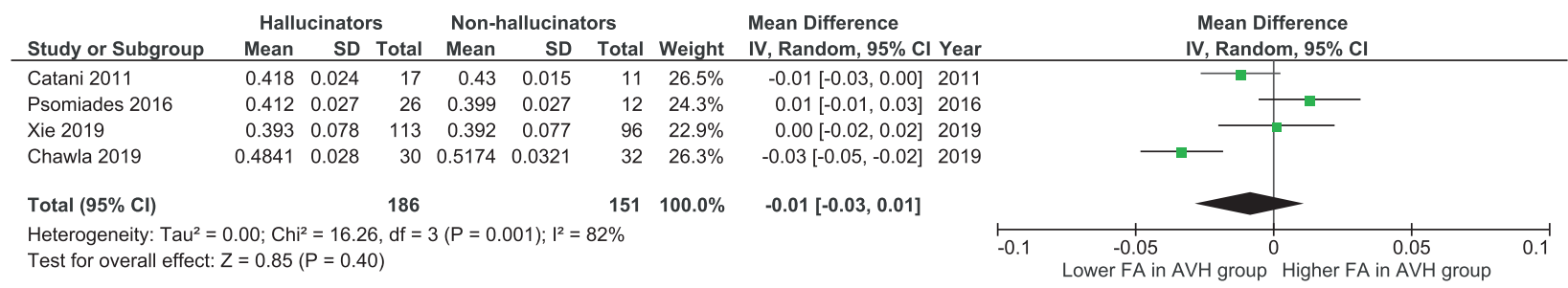

Fig. 5 A forest plot showing no significant difference between hallucinators and non-hallucinators for the fractional anisotropy of the right arcuate fasciculus. $S D$ standard deviation, $I V$ inverse variance, $\mathrm{Cl}$ confidence interval, $F A$ fractional anisotropy.

significantly reduced grey matter volume in hallucinators compared to non-hallucinators. Functional and structural abnormalities at the left insula may contribute to the misattribution of inner speech due to its suggested roles in both inner speech production and the salience network.

These contradictory findings and the remaining gaps in the evidence-base highlight that further exploration is required. This would be potentiated by further larger scale definitive primary research of inner speech production to confirm the regions involved in this process. Then, meta-analyses of trait studies should be prioritised to look for significant differences between hallucinators and non-hallucinators during inner speech production and selfreferential processing. Finally, additional novel meta-analyses could include using DTI metrics other than the FA to examine the arcuate fasciculi, as well as other language-related white matter tracts. Where possible: image-based meta-analyses should be preferred; nonhallucinators should have no lifetime experience of AVHs; hallucinators should have ongoing AVHs; anti-psychotic exposure should be minimal for both hallucinators and non-hallucinators.

\section{REFERENCES}

1. Zhang $X$, et al. Reduced white matter connectivity associated with auditory verbal hallucinations in first-episode and chronic schizophrenia: a diffusion tensor imaging study. Psychiatry Res Neuroimaging 2018;273:63-70.

2. Blom JD. Handbook of clinical neurology vol. 129 In: Aminoff MJ, Boller F, Swaab DF, editors. Auditory hallucinations. Ch. 24. New York: Elsevier; 2015.

3. Zmigrod L, Garrison JR, Carr J, Simons JS. The neural mechanisms of hallucinations: a quantitative meta-analysis of neuroimaging studies. Neurosci Biobehav Rev 2016;69:113-123. https://doi.org/10.1016/j.neubiorev.2016.05.037

4. Alderson-Day B, McCarthy-Jones S, Fernyhough C. Hearing voices in the resting brain: a review of intrinsic functional connectivity research on auditory verbal hallucinations. Neurosci Biobehav Rev 2015;55:78-87. https://doi.org/10.1016/j. neubiorev.2015.04.016

5. Jardri R, Pouchet A, Pins D, Thomas P. Cortical activations during auditory verbal hallucinations in schizophrenia: a coordinate-based meta-analysis. Am J Psychiatry 2011;168:73-81. https://doi.org/10.1176/appi.ajp.2010.09101522

6. Vilhauer RP. Depictions of auditory verbal hallucinations in news media. Int J Soc Psychiatry 2015;61:58-63. https://doi.org/10.1177/0020764014535757

7. van Lutterveld R, Diederen KMJ, Koops S, Begemann MJH, Sommer IEC. The influence of stimulus detection on activation patterns during auditory hallucinations. Schizophr Res 2013;145:27-32. https://doi.org/10.1016/j.schres.2013.01.004

8. Palaniyappan L, Balain V, Radua J, Liddle PF. Structural correlates of auditory hallucinations in schizophrenia: a meta-analysis. Schizophr Res 2012;137:169-173. https://doi.org/10.1016/j.schres.2012.01.038

9. Ćurčić-Blake B, et al. Interaction of language, auditory and memory brain networks in auditory verbal hallucinations. Prog Neurobiol 2017;148:1-20. https:// doi.org/10.1016/j.pneurobio.2016.11.002

10. Morin A, Michaud J. Self-awareness and the left inferior frontal gyrus: inner speech use during self-related processing. Brain Res Bull 2007;74:387-396. https://doi.org/10.1016/j.brainresbull.2007.06.013

11. Jones SR, Fernyhough C. Neural correlates of inner speech and auditory verbal hallucinations: a critical review and theoretical integration. Clin Psychol Rev 2007;27:140-154. https://doi.org/10.1016/j.cpr.2006.10.001

12. Hu C, et al. Distinct and common aspects of physical and psychological selfrepresentation in the brain: a meta-analysis of self-bias in facial and selfreferential judgements. Neurosci Biobehav Rev 2016;61:197-207. https://doi.org/ 10.1016/j.neubiorev.2015.12.003
13. Upthegrove $\mathrm{R}$, et al. Understanding auditory verbal hallucinations: a systematic review of current evidence. Acta Psychiatr Scand 2016;133:352-367. https://doi. org/10.1111/acps.12531

14. Kühn S, Gallinat J. Quantitative meta-analysis on state and trait aspects of auditory verbal hallucinations in schizophrenia. Schizophr Bull 2012;38:779-786. https://doi.org/10.1093/schbul/sbq152

15. Geoffroy PA, et al. The arcuate fasciculus in auditory-verbal hallucinations: a metaanalysis of diffusion-tensor-imaging studies. Schizophr Res 2014;159:234-237. https://doi.org/10.1016/j.schres.2014.07.014

16. Modinos G, et al. Neuroanatomy of auditory verbal hallucinations in schizophrenia: a quantitative meta-analysis of voxel-based morphometry studies. Cortex 2013;49:1046-1055. https://doi.org/10.1016/j.cortex.2012.01.009

17. Turkeltaub $P E$, et al. Minimizing within-experiment and within-group effects in activation likelihood estimation meta-analyses. Hum Brain Mapp 2012;33:1-13. https://doi.org/10.1002/hbm.21186

18. Fox PT, et al. User manual for GingerALE 2.3. http://www.brainmap.org/ale/ manual.pdf (2013).

19. Lancaster $\mathrm{JL}$, et al. Bias between $\mathrm{MNI}$ and talairach coordinates analyzed using the ICBM-152 brain template. Hum Brain Mapp 2007;28:1194-1205. https://doi. org/10.1002/hbm.20345

20. Fox M. BrainMap forums. http://www.brainmap.org/forum/viewtopic.php? $f=3 \& t=$ 608\&sid $=77$ c17593c9752103f11191e76d723e47 (2017).

21. Eickhoff SB, Bzdok D, Laird AR, Kurth F, Fox PT. Activation likelihood estimation meta-analysis revisited. Neuroimage 2012;59:2349-2361. https://doi.org/10.1016/ j.neuroimage.2011.09.017

22. Lancaster JL, Martinez MJ. Mango [computer program] version 4.1. Texas: Research Imaging Institute, UTHSCSA; 2019.

23. Chang $X$, et al. Distinct inter-hemispheric dysconnectivity in schizophrenia patients with and without auditory verbal hallucinations. Sci Rep. 2015;5:11218 https://doi.org/10.1038/srep11218

24. Review Manager (RevMan) [computer program] version 5.3. Copenhagen: The Nordic Cochrane Centre, The Cochrane Collaboration; 2014.

25. Fusar-Poli $P$, et al. Progressive brain changes in schizophrenia related to antipsychotic treatment? A meta-analysis of longitudinal MRI studies. Neurosci Biobehav Rev 2013;37:1680-1691. https://doi.org/10.1016/j.neubiorev.2013.06.001

26. Deng XY, Wang L, Yang TT, Li R, Yu G. A meta-analysis of diffusion tensor imaging of substantia nigra in patients with Parkinson's disease. Sci Rep. 2018;8:2941 https://doi.org/10.1038/s41598-018-20076-y

27. Sommer IE, et al. Auditory verbal hallucinations predominantly activate the right inferior frontal area. Brain 2008;131:3169-3177. https://doi.org/10.1093/brain/awn251

28. Diederen KMJ, et al. Deactivation of the parahippocampal gyrus preceding auditory hallucinations in schizophrenia. Am J Psychiatry 2010;167:427-435. https://doi.org/10.1176/appi.ajp.2009.09040456

29. Cierpka $M$, et al. Cerebellar contributions to persistent auditory verbal hallucinations in patients with schizophrenia. Cerebellum 2017;16:964-972. https://doi. org/10.1007/s12311-017-0874-5

30. Shapleske J, et al. A computational morphometric MRI study of schizophrenia: effects of hallucinations. Cereb Cortex 2002;12:1331-1341. https://doi.org/ 10.1093/cercor/12.12.1331

31. Gaser C, Nenadic I, Volz H, Büchel C, Sauer H. Neuroanatomy of "hearing voices": a frontotemporal brain structural abnormality associated with auditory hallucinations in schizophrenia. Cereb Cortex 2004;14:91-96. https://doi.org/10.1093/cercor/bhg107

32. Xie $\mathrm{S}$, et al. Hyperconnectivity in perisylvian language pathways in schizophrenia with auditory verbal hallucinations: A multi-site diffusion MRI study. Schizophr Res 2019;210:262-269. https://doi.org/10.1016/j.schres.2018.12.024

33. Leroux E, Delcroix N, Dollfus S. Abnormalities of language pathways in schizophrenia patients with and without a lifetime history of auditory verbal hallucinations: a DTI-based tractography study. World J Biol Psychiatry 2017;18:528-538. https://doi.org/10.1080/15622975.2016.1274053

34. Chawla N, Deep R, Khandelwal SK, Garg A. Reduced integrity of superior longitudinal fasciculus and arcuate fasciculus as a marker for auditory hallucinations 
in schizophrenia: a DTI tractography study. Asian J Psychiatr 2019;44:179-186. https://doi.org/10.1016/j.ajp.2019.07.043

35. Shergill SS, et al. Temporal course of auditory hallucinations. Br J 2004;185:516-7. https://doi.org/10.1192/bjp.185.6.516

36. Shergill SS, Bullmore E, Simmon A, Murray A, McGuire P. Functional anatomy of auditory verbal imagery in schizophrenic patients with auditory hallucinations. Am J Psychiatry 2000;157:1691-1693. https://doi.org/10.1176/appi.ajp.157.10.1691

37. Shergill SS, et al. A functional study of auditory verbal imagery. Psychol Med 2001;31:241-253. https://doi.org/10.1017/S003329170100335X

38. Palaniyappan L, Mallikarjun P, Joseph V, White TP, Liddle PF. Reality distortion is related to the structure of the salience network in schizophrenia. Psychol Med 2011;41:1701-1708. https://doi.org/10.1017/S0033291710002205

39. Wible CG, et al. $\mathrm{fMRI}$ activity correlated with auditory hallucinations during performance of a working memory task: data from the FBIRN Consortium Study. Schizophr Bull 2009;35:47-57. https://doi.org/10.1093/schbul/sbn142

40. Parellada $E$, et al. Fluordeoxyglucose-PET study in first-episode schizophrenic patients during the hallucinatory state, after remission and during linguisticauditory activation. Nucl Med Commun 2008;29:894-900. https://doi.org/ 10.1097/MNM.0b013e328302cd10

41. Cascella NG, Gerner GJ, Fieldstone SC, Sawa A, Schretlen DJ. The insula-claustrum region and delusions in schizophrenia. Schizophr Res 2011;133:77-81. https://doi. org/10.1016/j.schres.2011.08.004

42. Crick FC, Koch C. What is the function of the claustrum? Philos Trans R Soc Lond B Biol Sci 2005:360:1271-1279. https://doi.org/10.1098/rstb.2005.1661

43. Smith JB, et al. A role for the Claustrum in Salience Processing. Front Neuroanat 2019;13:64 https://doi.org/10.3389/fnana.2019.00064

44. Price CJ. The anatomy of language: a review of $100 \mathrm{fMRI}$ studies published in 2009. Ann N Y Acad Sci 2010;1191:62-88. https://doi.org/10.1111/j.1749-6632.2010.05444.x

45. Argyropoulos GP, Tremblay P, Small SL. The neostriatum and response selection in overt sentence production: an fMRI Study. Neuroimage 2013;18:53-60. https:// doi.org/10.1016/j.neuroimage.2013.05.064

46. Curčić-Blake B, et al. Not on speaking terms: hallucinations and structural network disconnectivity in schizophrenia. Brain Struct Funct 2015;220:407-418. https:// doi.org/10.1007/s00429-013-0663-y

47. Xi Y, et al. The structural connectivity pathology of first-episode schizophrenia based on the cardinal symptom of auditory verbal hallucinations. Psychiatry Res Neuroimaging 2016;257:25-30. https://doi.org/10.1016/j. pscychresns.2016.09.011

48. Salimi-Khorshidi G, Smith SM, Keltner JR, Wager TD, Nichols TE. Meta-analysis of neuroimaging data: a comparison of image-based and coordinate-based pooling of studies. Neuroimage 2009;45:810-823. https://doi.org/10.1016/j. neuroimage.2008.12.039

49. Eickhoff SB, et al. Behavior, sensitivity, and power of activation likelihood estimation characterized by massive empirical simulation. Neuroimage 2016;137:70-85. https://doi.org/10.1016/j.neuroimage.2016.04.072

50. Murray $\mathrm{R}$, et al. Should psychiatrists be more cautious about the long-term prophylactic use of antipsychotics? Br J Psychiatry 2016;209:361-365. https://doi. org/10.1192/bjp.bp.116.182683

51. Moher D, Liberati A, Tetzlaff J, Altman DG. The PRISMA Group. Preferred reporting items for systematic reviews and meta-analyses: The PRISMA statement. PLoS Med 2009;6:e1000097 https://doi.org/10.1371/journal.pmed.1000097

52. Silbersweig DA, et al. A functional neuroanatomy of hallucinations in schizophrenia. Nature 1995;378:176-179. https://doi.org/10.1038/378176a0

53. Lennox BR, Park SB, Medley I, Morris PG, Jones PB. The functional anatomy of auditory hallucinations in schizophrenia. Psychiatry Res 2000;100:13-20. https:// doi.org/10.1016/s0925-4927(00)00068-8

54. Copolov DL, et al. Cortical activation associated with the experience of auditory hallucinations and perception of human speech in schizophrenia: a PET correlation study. Psychiatry Res 2003;122:139-152. https://doi.org/10.1016/s09254927(02)00121-x

55. Hoffman RE, Anderson AW, Varanko M, Gore JC, Hampson M. Time course of regional brain activation associated with onset of auditory/verbal hallucinations Br. J Psychiatry 2008;193:424-425. https://doi.org/10.1192/bjp.bp.107.040501

56. Raij T, et al. Reality of auditory verbal hallucinations. Brain 2009;132:2994-3001. https://doi.org/10.1093/brain/awp186

57. Diederen KMJ, et al. Reproducibility of brain activation during auditory verbal hallucination. Schizophr Res 2013;146:320-325. https://doi.org/10.1016/j.schres.2013.01.025

58. Horga G, Schatz KC, Abi-Dargham A, Peterson BS. Deficits in predictive coding underlie hallucinations in schizophrenia. J Neurosci 2014;34:8072-8082. https:// doi.org/10.1523/JNEUROSCI.0200-14.2014

59. Thoma RJ, et al. Functional MRI evaluation of multiple neural networks underlying auditory verbal hallucinations in schizophrenia spectrum disorders. Front Psychiatry 2016;27:39 https://doi.org/10.3389/fpsyt.2016.00039
60. Neckelmann G, et al. Mr morphometry analysis of grey matter volume reduction in schizophrenia: Association with hallucinations. Int J Neurosci 2006;116:9-23. https://doi.org/10.1080/00207450690962244

61. O'Daly OG, Frangou S, Chitnis X, Shergill SS. Brain structural changes in schizophrenia patients with persistent hallucinations. Psychiatry Res 2007;156:15-21. https://doi.org/10.1016/j.pscychresns.2007.03.001

62. García-Martí G, et al. Schizophrenia with auditory hallucinations: a voxel-based morphometry study. Prog Neuro-Psychopharmacol Biol Psychiatry 2008;32:72-80. https://doi.org/10.1016/j.pnpbp.2007.07.014

63. Nenadic I, Smesny S, Schlösser RGM, Sauer H, Gaser C. Auditory hallucinations and brain structure in schizophrenia: voxel-based morphometric study. $\mathrm{Br} J$ Psychiatry 2010;196:412-413. https://doi.org/10.1192/bjp.bp.109.070441

64. van Tol MJ, et al. Voxel-based gray and white matter morphometry correlates of hallucinations in schizophrenia: the superior temporal gyrus does not stand alone. Neuroimage Clin 2014;4:249-257. https://doi.org/10.1016/j.nicl.2013.12.008

65. Seok J, et al. White matter abnormalities associated with auditory hallucinations in schizophrenia: a combined study of voxel-based analyses of diffusion tensor imaging and structural magnetic resonance imaging. Psychiatry Res 2007;156:93-104. https://doi.org/10.1016/j.pscychresns.2007.02.002

66. Catani $\mathrm{M}$, et al. Altered integrity of perisylvian language pathways in schizophrenia: relationship to auditory hallucinations. Biol Psychiatry 2011;70:1143-1150. https:// doi.org/10.1016/j.biopsych.2011.06.013

67. McCarthy-Jones S, Oestreich LKL, Whitford TJ. Reduced integrity of the left arcuate fasciculus is specifically associated with auditory verbal hallucinations in schizophrenia. Schizophr Res 2015;162:1-6. https://doi.org/10.1016/j.schres.2014.12.041

68. Psomiades $M$, et al. Integrity of the arcuate fasciculus in patients with schizophrenia with auditory verbal hallucinations: a DTI-tractography study. Neuroimage Clin 2016;12:970-975. https://doi.org/10.1016/j.nicl.2016.04.013

\section{ACKNOWLEDGEMENTS}

Xiangxin Liu for providing translation services in his spare-time. Anita Phul, Anna Cunningham, Rebecca Stephens and the rest of both the Barberry and Chesterfield Royal Hospital library teams for their help finalising the search strategy and accessing the full-texts of certain studies. This research did not receive any specific grant from funding agencies in the public, commercial, or not-for-profit sectors.

\section{AUTHOR CONTRIBUTIONS}

$R R$ and $R U$ developed the concept and aims of the manuscript. $L B$ conducted the literature search. All authors ( $L B, R R$ and $R U$ ) assessed the eligibility of studies. LB performed the data extraction and meta-analyses. Again, and finally, all authors (LB, $\mathrm{RR}, \mathrm{RU})$ contributed to the writing of the manuscript.

\section{COMPETING INTERESTS}

The authors declare no competing interests.

\section{ADDITIONAL INFORMATION}

Correspondence and requests for materials should be addressed to Liam Barber.

Reprints and permission information is available at http://www.nature.com/ reprints

Publisher's note Springer Nature remains neutral with regard to jurisdictional claims in published maps and institutional affiliations.

Open Access This article is licensed under a Creative Commons Attribution 4.0 International License, which permits use, sharing, adaptation, distribution and reproduction in any medium or format, as long as you give appropriate credit to the original author(s) and the source, provide a link to the Creative Commons license, and indicate if changes were made. The images or other third party material in this article are included in the article's Creative Commons license, unless indicated otherwise in a credit line to the material. If material is not included in the article's Creative Commons license and your intended use is not permitted by statutory regulation or exceeds the permitted use, you will need to obtain permission directly from the copyright holder. To view a copy of this license, visit http://creativecommons.org/licenses/by/4.0/.

(c) The Author(s) 2021 